and had been diagnosed with normocytic anemia. At that time, she received treatment with an iron preparation because of a low serum iron level despite her normal ferritin level. We estimate that the patient already had mild alveolar hemorrhage at that time and the bleeding later healed spontaneously. Among the cases of this disease reported from Japan, the disease tended to develop more frequently in the spring. In our patient, the disease developed twice (both times in June), suggesting the possibility that infection triggered the disease onset. Although alveolar hemorrhage subsided spontaneously in this case, lung fibrosis may develop in the future if alveolar bleeding develops repeatedly and its diagnosis is delayed. In the present case, treatment was started immediately after diagnosis and rapid aggravation of her renal function was prevented. When dealing with cases in whom unexplained normocytic anemia repeatedly occurs or cases with a discrepancy between serum iron and ferritin levels, the possibility of intrapulmonary bleeding should be considered and a chest X-ray should be obtained. Incidentally, after the diagnosis of this case was established, we inquired at the other hospital regarding the rapidly progressive glomerulonephritis of her younger sister and were informed that the disease was also confirmed to be ANCA-positive microscopic polyangiitis, identical to her elder sister's disease.

\section{References}

1 Ozen S, Ruperto N, Dillon MJ et al. EULAR/PReS endorsed consensus criteria for the classification of childhood vasculitides. Ann. Rheum. Dis. 2006; 65: 936-41.

2 Vanoni F, Bettinelli A, Keller F, Bianchetti MG, Simonetti GD. Vasculitides associated with IgG antineutrophil cytoplasmic autoantibodies in childhood. Pediatr. Nephrol. 2010; 25: 205-12.

3 Kobayashi S, Inokuma S. Intrapulmonary hemorrhage in collagenvascular disease includes a spectrum of underlying conditions. Intern. Med. 2009; 48: 891-7.

4 Hattori M, Kurayama H, Koitabashi Y. Antineutrophil cytoplasmic autoantibody-associated glomerulonephritis in children. J. Am. Soc. Nephrol. 2001; 12: 1493-500.

5 Peco-Antic A, Bonaci-Nikolic B, Basta-Jovanovic G et al. Childhood microscopic polyangiitis associated with MPO-ANCA. Pediatr. Nephrol. 2006; 21: 46-53.

$6 \mathrm{Yu}$ F, Huang J-P, Zou W-Z, Zhao M-H. The clinical features of anti-neutrophil cytoplasmic antibody-associated systemic vasculitis in Chinese children. Pediatr. Nephrol. 2006; 21: 497-502.

7 Yang F, Haile DJ, Berger FG, Herbert DC, Beveren EV, Ghio AJ. Haptoglobin reduces lung injury associated with exposure to blood. Am. J. Physiol. Lung Cell. Mol. Physiol. 2003; 284: L402-9.

8 Yao T-C, Hung I-J, Jaing T-H, Young C-P. Pitfalls in the diagnosis of idiopathic pulmonary haemosiderosis. Arch. Dis. Child. 2002; 86: 436-9.

\title{
Hyper-eosinophilia in granular acute B-cell lymphoblastic leukemia with myeloid antigen expression
}

\author{
Daisuke Kobayashi, ${ }^{1,2}$ Kazuhiro Kogawa, ${ }^{2}$ Kohsuke Imai, ${ }^{2}$ Toshio Tanaka, ${ }^{3}$ Akiko Sada ${ }^{4}$ and Shigeaki Nonoyama ${ }^{2}$ \\ ${ }^{1}$ Carman and Ann Adams Department of Pediatrics, Children's Hospital of Michigan, Wayne State University School of \\ Medicine, Detroit, Michigan, USA, ${ }^{2}$ Department of Pediatrics, National Defense Medical College, Tokorozawa, Saitama, \\ ${ }^{3}$ Graduate School of Health Care Sciences, Tokyo Medical and Dental University, Tokyo and ${ }^{4}$ Department of Internal \\ Medicine, Kobe University, Kobe, Hyogo, Japan
}

Abstract Acute lymphoblastic leukemia with eosinophilia (ALLEo) is a rare but a distinctive clinical entity. Clinical features of idiopathic hyper-eosinophilic syndrome (HES) can be seen in patients with ALLEo. We report a 10-year-old girl, in whom HES was initially suspected but further investigation confirmed the diagnosis of acute B-cell lymphoblastic leukemia with myeloid antigen expression. Clinical response to chemotherapy was excellent with achievement of complete remission for 4 years. Serum interleukin-3 and -5 were elevated at presentation and normalized with disappearance of eosinophilia after induction therapy, supporting the reactive nature of eosinophilia in ALLEo. Hematologic malignancy should be considered in patients with hyper-eosinophilia, before attributing it to HES.

Key words acute lymphoblastic leukemia, eosinophilia, idiopathic hyper-eosinophilic syndrome, interleukin-3, interleukin-5, myeloid antigen.

Correspondence: Daisuke Kobayashi, MD, Carman and Ann Adams Department of Pediatrics, Children's Hospital of Michigan, Wayne State University School of Medicine, 3901 Beaubien Boulevard, Detroit, MI 48201-2119, USA. E-mail: dkobayas@dmc.org

Received 23 February 2011; revised 24 June 2011; accepted 29 August 2011.

doi: 10.1111/j.1442-200X.2011.03471.x
Acute lymphoblastic leukemia with eosinophilia (ALLEo) is a rare but distinctive clinical entity. There have been approximately 50 cases of ALLEo reported. ${ }^{1-4}$ Patients with ALLEo can present with clinical features of idiopathic hyper-eosinophilic syndrome (HES) due to eosinophilic organ infiltration. Bone marrow analysis is important for investigating the etiology of eosinophilia even 
without blasts in peripheral blood smear or cytopenia. ${ }^{5}$ Further diagnostic investigations to exclude different types of hematologic malignancy are important before attributing peripheral blood eosinophilia to HES. ${ }^{6}$ Herein we describe the case of a 10-year-old girl with acute B-cell lymphoblastic leukemia with myeloid antigen expression, in whom cytokine production in the lymphoblasts was investigated.

\section{Case report}

A 10-year-old girl developed pruritic, erythematous rash on her extremities, 6 months prior to presentation. One month prior to presentation, she had persistent skin rash and headache with an absolute eosinophil count of $8.93 \times 10^{9} / \mathrm{L}$. She was then admitted to the National Defense Medical College hospital because of arthralgia in both wrists, swelling of right shoulder, and shortness of breath with exertion. Her past and family history was unremarkable. On admission her vital signs were within normal limits, with normal cardiopulmonary examination. On complete blood count the white blood cells (WBC) were $12.6 \times 10^{9} / \mathrm{L}$, hemoglobin was $12.0 \mathrm{~g} / \mathrm{dL}$, and the platelets were $177 \times 10^{9} / \mathrm{L}$ with differential of neutrophilic bands (35\%), neutrophilic segmentals (9\%), lymphocytes (15\%), and eosinophils (39\%). There were no blasts on the peripheral blood smear. Eosinophils were hyperlobulated with decreased cytoplasmic granules (Fig. 1a).

We investigated the etiology of eosinophilia and found elevated C-reactive protein of $3.5 \mathrm{mg} / \mathrm{dL}$ and erythrocyte sedimentation rate of $50 \mathrm{~mm} / \mathrm{h}$. Soluble interleukin-2 (IL-2) receptor was elevated at $4450 \mathrm{U} / \mathrm{mL}$. Ova and parasite study of stool was negative. Chest radiography and computed tomography of the chest showed diffuse infiltrates in both lungs. The pulmonary function test showed a restrictive pattern. Echocardiography showed good biventricular function. We suspected HES, because the patient had multiple organ involvement with significant eosinophilia.

Bone marrow aspiration was performed to exclude hematologic malignancy and indicated hypercellular marrow with lymphoblasts $90 \%$ and eosinophils $2 \%$. Lymphoblasts were L1 in the French-American-British classification and were negative on myeloperoxidase and periodic acid-Schiff stain (Fig. 1b). Cytoplasmic granules of lymphoblasts were positive for $\alpha$ naphthyl-butyrate esterase without $\mathrm{NaF}$ inhibition (Fig. 1c). Immunophenotyping on bone marrow CD45 dim cells showed lymphoblastic B-lineage cells expressing CD10, CD19, CD20, CD22, CD34, CD38, and the myeloid antigen CD33; this confirmed the diagnosis of acute precursor B-cell lymphoblastic leukemia. Cytogenetic analysis indicated normal karyotype 46,XX. Cerebrospinal fluid was negative for leukemia (1 WBC, 0 red blood cells, no blasts). The patient was enrolled in the Children's Cancer and Leukemia Study Group protocol ALL2004 (high-risk arm). Induction chemotherapy was initiated with adriamycin, vincristine, prednisone, and L-asparginase. Her symptoms, pulmonary infiltrates, and eosinophilia resolved quickly with induction chemotherapy, and the patient has remained in complete remission for 4 years since the onset of the disease.

We measured levels of IL-3, IL-5, and granulocyte macrophage-colony-stimulating factor (GM-CSF) in serum,
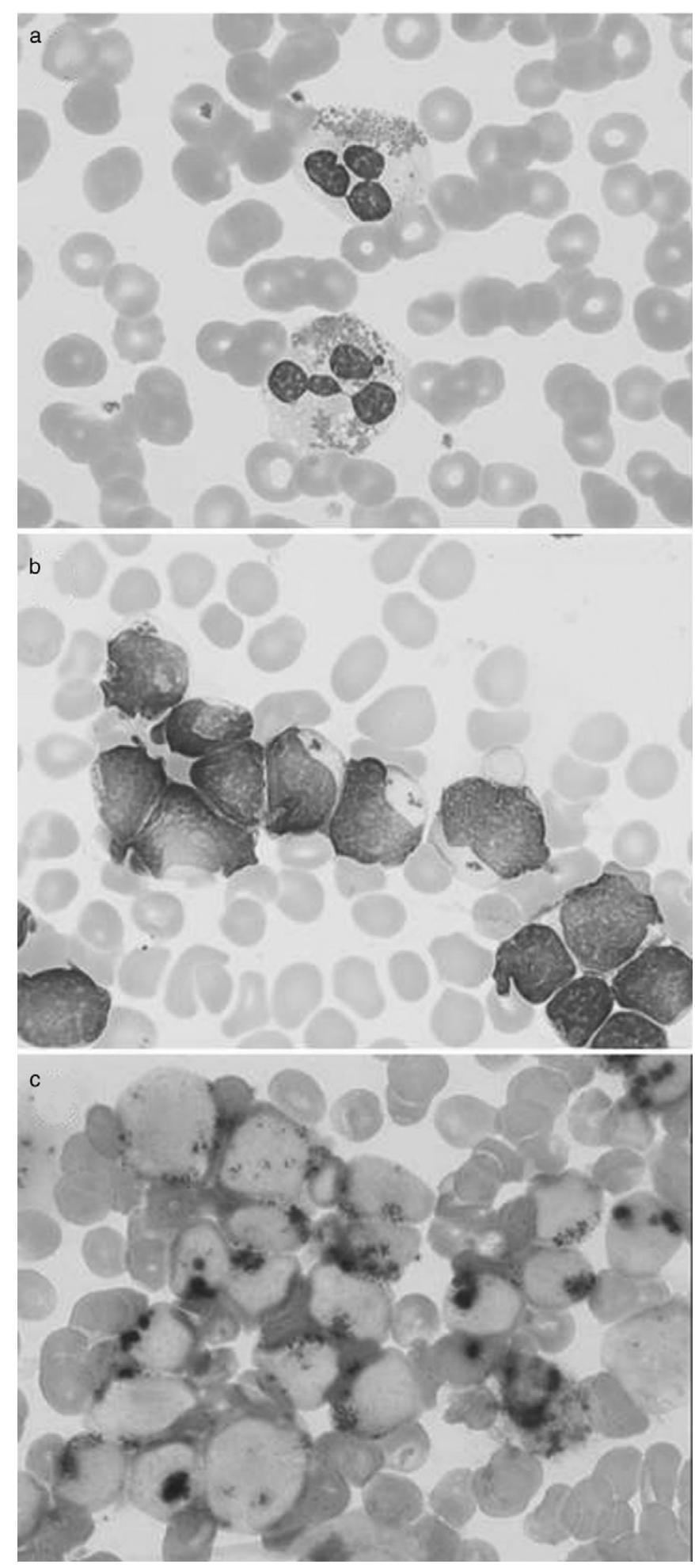

Fig. 1 Pathology on admission. (a) Eosinophils on peripheral blood smear. Eosinophils were hyperlobulated with decreased cytoplasmic granules. (b) Lymphoblasts in bone marrow. Wright-Giemsa stain. (c) Lymphoblasts in bone marrow. Cytoplasmic granules positive for $\alpha$-naphthyl-butyrate esterase stain. Granules were not inhibited by $\mathrm{NaF}$.

\section{(C) 2012 The Authors}

Pediatrics International (C) 2012 Japan Pediatric Society 
Table 1 Cytokine assay

\begin{tabular}{lccc}
\hline & $\begin{array}{c}\text { On } \\
\text { admission }\end{array}$ & $\begin{array}{c}\text { After induction } \\
\text { chemotherapy }\end{array}$ & $\begin{array}{c}\text { Reference } \\
\text { level }\end{array}$ \\
\hline Interleukin-3 $(\mathrm{pg} / \mathrm{mL})$ & 95 & $\leq 31$ & $\leq 31$ \\
Interleukin-5 $(\mathrm{pg} / \mathrm{mL})$ & 29.8 & 10.2 & $\leq 10$ \\
GM-CSF $(\mathrm{pg} / \mathrm{mL})$ & $\leq 8$ & $\leq 8$ & $\leq 8$ \\
\hline
\end{tabular}

Granulocyte macrophage-colony-stimulating factor.

using an ELISA kit (IL-3, GM-CSF: R\&D Systems, Minneapolis, MN, USA; IL-5: Immunotech, Marseille, France). IL-3 and IL-5 in serum were elevated on admission, and normalized after induction therapy (Table 1). We assayed the expression of Fip1-like1-platelet-derived growth factor receptor alpha (FIP1L1-PDGFRA) mRNA on reverse transcriptase-polymerase chain reaction (RT-PCR) using the lymphoblasts in bone marrow, as previously described. ${ }^{7}$ RT-PCR of FIP1L1-PDFGRA mRNA was not detected in this case.

\section{Discussion}

Acute lymphoblastic leukemia with eosinophilia occurs rarely and 52 cases of ALLEo have been reported in 44 studies. ${ }^{7,8} \mathrm{~A}$ review of 38 cases of ALLEo reported male predominance (76\%), a median age of 14 years, and a median absolute eosinophil count at presentation of $10.78 \times 10^{9} / \mathrm{L}$ as well as clinical presentation such as splenomegaly $(60 \%)$, skin rash $(54 \%)$, cough or dyspnea (43\%), pneumonia or pulmonary infiltrates $(51 \%)$, chest pain $(20 \%)$, and adenopathy $(46 \%){ }^{2}$ Skin findings reported in 14 cases of ALLEo are diverse, including rash, purpura, ecchymosis, and one report of erythroderma. ${ }^{3}$ Eosinophilia may antedate the development of ALL by several months to 2 years, and patients may present features of HES during this period. ${ }^{9}$ Eosinophilia resolves with remission and usually returns with a relapse of the disease. ${ }^{10}$ The majority of cases of ALLEo are of the B-cell type, and a few cases have been described of T-cell ALLEo; B-cell ALLEo with myeloid antigen expression; and biphenotypic (B-cell/T-cell) ALLEo. ${ }^{1,5,10}$ Granular ALL confers a poor prognosis on children with ALL of the B-cell precursor phenotype, ${ }^{11}$ and two cases of granular ALLEo have been described. ${ }^{5,12}$ Cytogenetic abnormalities have been detected in approximately half (30/52) of the patients with ALLEo. ${ }^{4}$ The most common abnormality has been $\mathrm{t}(5 ; 14)(\mathrm{q} 31 ; \mathrm{q} 32)$. This entity is now included as "B-lymphoblastic leukemia/lymphoma with $\mathrm{t}(5 ; 14)$; IL-3-IGH" in the new World Health Organization classification of lymphoid neoplasms published in 2008. The present case was a rare form of B-cell ALLEo with granular lymphoblasts, myeloid antigen expression, and normal cytogenetic testing. The present patient has remained in remission for 4 years, and her symptoms, pulmonary infiltrates, and eosinophilia responded quickly to induction chemotherapy.

Eosinophilia is considered to be reactive in ALLEo and results from cytokine production by leukemic blasts (because chromosomal abnormality is usually found only in leukemic blasts); and eosinophils have normal karyotypes in $90 \%$ of cases. ${ }^{4}$ In ALLEo with $\mathrm{t}(5 ; 14)(\mathrm{q} 31: \mathrm{q} 32)$, eosinophilia is secondary to overproduction of IL-3 by the blasts due to activation of the IL-3 gene on chromosome 5 after its translocation, adjacent to the immunoglobulin heavy chain gene on chromosome $14 .{ }^{8}$ In the present patient, the level of IL-3 and IL-5 in the serum was significantly elevated on admission and normalized with treatment. IL-3 and IL- 5 were likely to be produced by the lymphoblasts. The present finding further supports the assumption that eosinophilia arises due to cytokine production by leukemic blasts in patients with ALLEo. We cannot, however, exclude the possibility that activated leukocytes including eosinophils produce a large amount of cytokines. In addition, chemotherapy reduces normal hematopoietic cells as well as leukemic cells.

The FIP1L1-PDGFRA fusion has been described in patients with chronic eosinophilic leukemia, which can present as idiopathic HES. Recent report showed that the presence of the FIP1L1-PDGFRA fusion gene was detected in five patients with acute myeloid leukemia and associated eosinophilia. ${ }^{13}$ In these cases, FIP1L1-PDGFRA-positive cells were seen in $>90 \%$ of all marrow cells, clearly indicating that blast cells were indeed FIP1L1-PDGFRA positive. The presence of FIP1L1-PDGFRA was confirmed in all these cases by amplification and sequencing of the RNA fusion sequences on RT-PCR and of the genomic DNA fusion sequences on long-template-PCR and bubble-PCR. In the present case, we investigated the presence of FIP1L1PDGFRA mRNA in lymphoblasts in bone marrow, because this fusion gene was reported in the blasts of acute myeloid leukemia.

In conclusion, we describe a rare form of granular B-cell ALLEo with myeloid antigen expression. Eosinophilia disappeared along with the normalization of IL-3 and IL-5 in the serum on chemotherapy; it further supports the reactive nature of eosinophilia in ALLEo. FIP1L1-PDGFRA was negative, and clinical response to chemotherapy was excellent with achievement of complete remission for 4 years. Hematologic malignancy should be considered in every patient with hyper-eosinophilia, before attributing it to HES.

\section{Acknowledgments}

The authors would like to thank Ms Makiko Tanaka, National Defense Medical College, Saitama, Japan, for her skillful assistance. This work was supported by a grant from the Ministry of Health, Labor, and Welfare of Japan; Japan Defense Agency; and by a grant from Kawano Masanori Foundation for Promotion of Pediatrics.

\section{References}

1 Wilson F, Tefferi A. Acute lymphocytic leukemia with eosinophilia: Two case reports and a literature review. Leuk. Lymphoma 2005; 46: 1045-50.

2 Fishel RS, Farnen JP, Hanson CA, Silver SM, Emerson SG. Acute lymphoblastic leukemia with eosinophilia. Medicine 1990; 69: 232-43.

3 Chien AJ, Argenyi ZB, Colven RM, Kirby P. Acute lymphoblastic leukemia presenting with urticarial plaques and hypereosinophilia in a child. J. Am. Acad. Dermatol. 2004; 51: S151-5.

4 Sutton R, Lonergan M, Tapp H et al. Two cases of hypereosinophilia and high-risk acute lymphoblastic leukemia. Leukemia 2008; 22: $1463-5$. 
5 Girodon F, Bergoin E, Favre B et al. Hypereosinophilia in acute B-lineage lymphoblastic leukaemia. Br. J. Haematol. 2005; 129: 568.

6 Hagle KT, Zori RT, Yuan CM, Gray BA, Moreb JS. Diagnosis of an early precursor-B-ALL presenting with hypereosinophilia using FISH on immunomagnetically selected CD19+ cells. Clin. Adv. Hematol. Oncol. 2005; 3: 62-4.

7 Sada A, Katayama Y, Yamamoto K et al. Japanese Elderly Leukemia and Lymphoma Study Group. A multicenter analysis of the FIP1L1-alphaPDGFR fusion gene in Japanese idiopathic hypereosinophilic syndrome: An aberrant splicing skipping the alphaPDGFR exon 12. Ann. Hematol. 2007; 86: 855-63.

8 Grimaldi JC, Meeker TC. The t $(5 ; 14)$ chromosomal translocation in a case of acute lymphocytic leukemia joins the interleukin-3 gene to the immunoglobulin heavy chain gene. Blood 1989; 73: 2081-5.

9 Hill A, Metry D. Urticarial lesions in a child with acute lymphoblastic leukemia and eosinophilia. Pediatr. Dermatol. 2003; 20: $502-5$.
10 Narayanan G, Hussain BM, Chandralekha B, Sivasankar C, Nair MK. Hypereosinophilic syndrome in acute lymphoblastic leukaemia: Case report and literature review. Acta Oncol. 2000; 39: 241-3.

11 Cerezo L, Shuster JJ, Pullen DJ et al. Laboratory correlates and prognostic significance of granular acute lymphoblastic leukemia in children. A Pediatric Oncology Group study. Am. J. Clin. Pathol. 1991; 95: 526-31.

12 Jain P, Kumar R, Gujral S et al. Granular acute lymphoblastic leukemia with hypereosinophilic syndrome. Ann. Hematol. 2000; 79: 272-4.

13 Metzgeroth G, Walz C, Score J et al. Recurrent finding of the FIP1L1-PDGFRA fusion gene in eosinophilia-associated acute myeloid leukemia and lymphoblastic T-cell lymphoma. Leukemia 2007; 21: 1183-8.

\title{
Atomoxetine improves communication in a girl with semantic-pragmatic disorder
}

\author{
Kayo Inoko, Kayano Kodaira and Makiko Osawa \\ Department of Pediatrics, Tokyo Women's Medical University, Tokyo, Japan
}

\begin{abstract}
This report describes the case of an ADHD girl (hereafter referred to as $\mathrm{K}$ ) with semantic-pragmatic disorder, she was treated with atomoxetine. K was a 9-year-old girl. She had difficulty understanding words or sentences, finding words, and producing sentences. K also displayed symptoms of severe inattentiveness. K was diagnosed with DSM-IV-defined ${ }^{1}$ ADHD, predominantly the inattentive type. Her communication impairment was considered symptomatic of semanticpragmatic disorder. $\mathrm{K}$ was started on atomoxetine, the dose was increased to $50 \mathrm{mg} /$ day (dosage based on weight: $1.8 \mathrm{mg} / \mathrm{kg}$ ). Her communication activities were improved a few weeks after atomoxetine $50 \mathrm{mg} /$ day was administered. She was unable to organize information pertaining to words, and so could not use words in expressive language. These problems were mitigated through the administration of atomoxetine. Further prospective studies are needed to better understand the therapeutic effects of atomoxetine in patients with semantic-pragmatic disorder.
\end{abstract}

Key words atomoxetine, attention-deficit hyperactivity disorder, executive function, semantic-pragmatic disorder.

Attention-deficit hyperactivity disorder (ADHD) is characterized by behaviors of inattentiveness, hyperactivity and impulsivity. ${ }^{1}$ Neuropsychological impairments may accompany the behavioral manifestations, and deficits in executive function are evident in many children and adolescents with ADHD. Atomoxetine hydrochloride has been indicated for the treatment of ADHD. Cognition may be related to functional outcomes, and atomoxetine improves attention and executive function as well as other cognitive domains. ${ }^{2}$ Many children with ADHD have comorbid diagnoses $, 3,4$ and behavioral problems associated with ADHD are

Correspondence: Kayo Inoko, MD, Inoko Mental Clinic, 2-70 Hanasaki-cho, Naka-ku, Yokohama 231-0063, Japan. Email: inokomental@nifty.com

Received 8 November 2010; revised 18 October 2011; accepted 6 December 2011.

doi: 10.1111/j.1442-200X.2012.03581.x related to pragmatic competence. ${ }^{5}$ Pragmatic difficulties involve secondary consequences of specific language impairments, and disproportionate pragmatic difficulties in mastering structural aspects of language are termed semantic-pragmatic disorder. ${ }^{6}$ Some children with semantic-pragmatic disorder have limited language structures, and the pragmatic difficulties may be regarded as secondary to the structural language difficulties; in others, phonology and syntax are unimpaired, and the pragmatic difficulties may be regarded as an indication that the child has autism or an autistic spectrum disorder. There may be many intermediate cases. ${ }^{6}$ Children with semantic-pragmatic disorder have greater difficulty in making conversation than the limitations of language structure can explain. Semantic-pragmatic disorder without impairment of phonology or syntax is characterized by verbosity with comprehension deficits for connected speech, word-finding deficits and atypical word choice frequent, 